\begin{tabular}{|c|c|c|}
\hline \multirow{3}{*}{$\begin{array}{r}\text { Case Reports in } \\
\text { Gastroenterology }\end{array}$} & \multirow{2}{*}{\multicolumn{2}{|c|}{ Case Rep Gastroenterol 2014;8:227-234 }} \\
\hline & & \\
\hline & $\begin{array}{l}\text { DOI: } 10.1159 / 000365320 \\
\text { Publisnea onilne: June } \angle 6,2014\end{array}$ & $\begin{array}{l}\text { (c) } 2014 \text { S. Karger AG, Basel } \\
\text { 1662-0631/14/0082-0227\$39.50/0 } \\
\text { www.karger.com/crg }\end{array}$ \\
\hline & \multicolumn{2}{|c|}{$\begin{array}{l}\text { This is an Open Access article licensed under the terms of the Creative Commons } \\
\text { Attribution-NonCommercial } 3.0 \text { Unported license (CC BY-NC) (www.karger.com/OA } \\
\text { license), applicable to the online version of the article only. Distribution permitted for non } \\
\text { commercial purposes only. }\end{array}$} \\
\hline
\end{tabular}
commercial purposes only.

\title{
Rapidly Growing Esophageal Carcinosarcoma Reduced by Neoadjuvant Radiotherapy Alone
}

\author{
Naotaka Ogasawara ${ }^{a}$ Yasuhiro Tamura ${ }^{a} \quad$ Yasushi Funaki $^{a}$ \\ Yoshiharu Yamaguchi $^{\text {a }}$ Akihiro Shimozato $^{\text {a }}$ Kenichiro Yanamoto $^{a}$ \\ Emiko Takahashi $^{b}$ Masahiko Miyachic Makoto Sasaki ${ }^{a}$ Kunio Kasugai $^{a}$ \\ Departments of a Gastroenterology, ${ }^{b}$ Pathology and ${ }^{\mathrm{c}}$ Surgery, Aichi Medical University \\ School of Medicine, Nagakute, Japan
}

\section{Key Words}

Dysphagia - Gastrointestinal cancer - Malignant transformation - Squamous cell carcinoma .

Treatment

\begin{abstract}
Esophageal carcinosarcoma is a rare malignant neoplasm consisting of both carcinomatous and sarcomatous components. It is generally treated by surgery, radiotherapy and chemotherapy according to the protocols used for other esophageal cancers. However, the treatment of esophageal carcinosarcoma by radiotherapy alone before surgery has not been previously described. We report a patient with a rapidly growing esophageal carcinosarcoma that was efficiently reduced by neoadjuvant radiotherapy alone. A previously healthy 69year-old man was admitted with dysphagia. Initial esophagogastroduodenoscopy (EGD) revealed a small nodular polypoid lesion of about $10 \mathrm{~mm}$ in the middle esophagus. A second EGD 1 month later showed that the tumor had expanded into a huge mass. A biopsy specimen revealed that the tumor comprised squamous cell carcinoma with spindle cell components, and the tumor was diagnosed as carcinosarcoma which was diagnosed as stage I (T1bNOM0). Due to renal dysfunction, the patient was treated with neoadjuvant radiotherapy (40 Gy) without chemotherapy. A third EGD 1 month later revealed remarkable tumor reduction. He then underwent total esophagectomy with regional lymph node dissection (pStage 0, pT1aNOM0). After surgical operation, the patient was followed up without adjuvant therapy. Whole body computed tomography revealed lung metastasis 14 months after surgery, and the patient died 2 months later. The neoadjuvant radiotherapy for esophageal carcinosarcoma was considered to have contributed to the subsequent surgery and his pro-
\end{abstract}

Naotaka Ogasawara, MD

Department of Gastroenterology

Aichi Medical University School of Medicine

1-1 Yazakokarimata, Nagakute, Aichi 480-1195 (Japan)

E-Mail nogasa@aichi-med-u.ac.jp 
Ogasawara et al.: Rapidly Growing Esophageal Carcinosarcoma Reduced by Neoadjuvant Radiotherapy Alone

longed survival time. Thus, radiotherapy alone might be a suitable neoadjuvant therapy for esophageal carcinosarcomas.

(c) 2014 S. Karger AG, Basel

\section{Introduction}

Esophageal cancer is the third most common gastrointestinal cancer [1]. While 89\% of reported esophageal cancers are of the squamous cell type, only $1.3 \%$ of them present as carcinosarcomas [1]. Carcinosarcoma of the esophagus is a rare malignant neoplasm consisting of both carcinomatous and sarcomatous components. Although the oncogenesis of carcinosarcoma remains unclear, both metaplastic and collision concepts have been proposed. The former involves individual elements derived from a single common ancestor cell (so-called carcinosarcoma), and the latter involves two individual stem cells that independently and simultaneously undergo malignant transformation (true carcinosarcoma) [2]. The 5-year survival rates after treatment are quite similar to those reported for squamous cell carcinoma (SCC) $[3,4]$. Although the 3-year survival rate is higher for carcinosarcoma than for SCC, the 5-year survival rate does not significantly differ between them. Esophageal carcinosarcoma is usually treated by partial or total esophagectomy with regional lymph node dissection according to the protocols used for other esophageal cancers [3-5]. A few reports have described radiotherapy and chemotherapy for esophageal carcinosarcoma before or after surgery. One study found that radiotherapy could not control recurrent tumor growth after esophagectomy [5], whereas another found that chemotherapy and radiotherapy reduced primary lesions by $45 \%$ after surgery including an esophageal bypass with a stomach tube [6]. However, the effects of treating esophageal carcinosarcoma by neoadjuvant radiotherapy before surgery have not been described.

We describe a patient with a rapidly growing esophageal carcinosarcoma that was efficiently reduced by radiotherapy alone before surgical treatment.

\section{Case Report}

A previously healthy 69-year-old man was admitted to our hospital with dysphagia. His family and medical histories were unremarkable. Neither lymphadenopathy nor thyroid swelling were evident and abdominal findings were normal. All laboratory data upon admission were within normal limits except for creatinine, which was $1.6 \mathrm{mg} / \mathrm{dl}$. Creatinine clearance was estimated as $33.9 \mathrm{ml} / \mathrm{min}$. Tumor marker levels were all within normal limits: SCC antigen $1.3 \mathrm{ng} / \mathrm{ml}$, carcinoembryonic antigen $1.9 \mathrm{ng} / \mathrm{ml}$ and carbohydrate antigen 19-9 $11 \mathrm{U} / \mathrm{l}$. A first esophagogastroduodenoscopy (EGD) revealed a small nodular polypoid lesion about $10 \mathrm{~mm}$ in diameter with central ulceration in the lower esophagus (fig. 1a). The tumor surface was not stained by Lugol's iodine (fig. 1b). A second EGD 1 month later showed that the tumor had expanded into a huge mass that occupied the esophageal lumen (fig. 1c). The tumor surface was relatively smooth and fuzzy (fig. 1c) and part of the surface comprised normal esophageal epithelial mucosa (fig. 1d, arrows). However, due to the size of the tumor the basal part could not be visualized by EGD. Contact with EGD caused slight oozing from the surface of the tumor. The esophageal mucosa surrounding the tumor was rough (fig. 1a) and not stained by Lugol's iodine (fig. 1b). However, biopsy specimens obtained from the esophageal mucosa surrounding the tumor revealed no malignancy. Moreover, 1 month of treatment with a proton pump inhibitor essentially normalized the esophageal mucosa surrounding the tumor (fig. 1c, d). Thus, we considered that gastroesophageal reflux of the 
Ogasawara et al.: Rapidly Growing Esophageal Carcinosarcoma Reduced by Neoadjuvant Radiotherapy Alone

stomach contents had caused the transition of the surrounding esophageal mucosa. Esophageal ultrasonography revealed that the tumor was internally hypoechoic and relatively homogeneous (fig. 1e). The fourth layer was obvious (fig. 1e, arrowheads), and we thus considered that the tumor had not invaded the muscularis propria. Barium esophagography revealed a lobulated polypoid lesion about $6 \mathrm{~cm}$ in diameter with a relatively smooth surface, a central superficial irregularity and depression in the lower esophagus (fig. 2a). A biopsy specimen showed that the tumor comprised poorly differentiated SCC with spindle cell components (fig. 3a). Immunohistochemical evaluation of a biopsy specimen of the tumor obtained at the time of the first EGD revealed that the SCC cells were positive for cytokeratin (fig. 3b). In contrast, the spindle-shaped tumor cells were positive for vimentin and negative for cytokeratin (fig. 3c), CD34 (fig. 3d) and $\alpha$-smooth muscle actin. Transitional features between the two components were also evident. The tumor was finally diagnosed as carcinosarcoma. Whole body computed tomography found no evidence of metastasis. According to the Guidelines for Clinical and Pathologic Studies on Carcinoma of the Esophagus, 10th edition [7], the esophageal carcinosarcoma was diagnosed as stage I (T1bN0M0).

At that time, the patient refused surgical treatment but accepted chemoradiotherapy. Due to renal dysfunction, he was treated with neoadjuvant radiotherapy (40 Gy) alone without chemotherapy. A third EGD 1 month after radiotherapy showed a remarkable reduction in the size of the tumor (fig. 1f), which was visualized as a small polypoid lesion. Barium esophagography also revealed a tumor about $1 \mathrm{~cm}$ in diameter with a smooth surface (fig. 2b). These findings indicated that neoadjuvant radiotherapy alone had been exceedingly effective for carcinosarcoma. He finally agreed to surgical treatment and underwent total esophagectomy with regional lymph node dissection. Pathological examination revealed that the esophageal carcinosarcoma had invaded the lamina propria mucosa and that resected regional lymph nodes harbored no malignancy. At that time, the esophageal carcinosarcoma was finally diagnosed as pStage 0 (pT1aN0M0). After surgical operation, the patient was followed up as an outpatient without adjuvant therapy. Whole body computed tomography revealed bilateral lung metastasis 14 months later. However, the patient refused treatment for the metastasis and died 16 months after surgery.

\section{Discussion}

Composite malignant tumors with both carcinomatous and sarcomatous components are known as carcinosarcomas. Esophageal carcinosarcoma reportedly accounts for 0.47$2.4 \%$ of all esophageal tumors $[4,8,9]$. Virchow [10] in 1865 investigated a rare malignant neoplasm consisting of carcinomatous and sarcomatous components and referred to it as carcinosarcoma. Since then, such neoplasms have also been referred to as sarcomatoid carcinoma, pseudosarcoma or spindle cell carcinoma. In 1992, Ro et al. [11] proposed the following histological criteria for carcinosarcoma: concurrent malignant epithelial and spindle cell components with transitional areas in between and a sarcomatoid component expressing an epithelial phenotype. According to these criteria, the Guidelines for Clinical and Pathological Studies on Carcinoma of the Esophagus, 9th edition classified esophageal carcinosarcoma as so-called carcinosarcoma, pseudosarcoma and true carcinosarcoma [12]. However, a recent report found no clinical or behavioral differences among the subtypes [3]. Thus, analysis of the characteristics of esophageal carcinosarcoma as a whole is important. Therefore, a classification of subtypes might not be essential for considering the clinical outcomes and features of carcinosarcoma, and the tumor in our patient was classified as 
Ogasawara et al.: Rapidly Growing Esophageal Carcinosarcoma Reduced by Neoadjuvant Radiotherapy Alone

esophageal carcinosarcoma according to the Guidelines for Clinical and Pathologic Studies on Carcinoma of the Esophagus, 10th edition [7].

Among 87 patients with carcinosarcoma described in the literature, 83 (95.4\%), 3 (3.4\%) and $1(1.1 \%)$ were treated by surgical resection, local excision and endoscopic resection, respectively. Regional lymph nodes were involved in 31 of 59 patients (52.5\%) [3, 13]. Another review of 57 patients with esophageal carcinosarcoma described that 54 patients $(94.7 \%)$ were treated by curative resection, of whom $15(27.8 \%)$ received additional treatment, including chemotherapy $(n=8)$, chemoradiotherapy $(n=6)$ and radiotherapy alone $(n=1)$ [6]. Sanada et al. [6] reported that chemotherapy (5-fluorouracil plus docetaxel) and radiotherapy (66 Gy) for 2 months achieved a $45 \%$ reduction rate for primary lesions after surgery comprising an esophageal bypass with a stomach tube. On the other hand, Iwaya et al. [5] reported that radiotherapy could not control recurrent tumor growth after esophagectomy. In the review by Sanada et al. [6], only 2 patients received neoadjuvant chemoradiotherapy before surgery. These two reports are published in the Japanese literature and cannot be found by searching PubMed (National Library of Medicine). However, esophageal carcinosarcoma treated by radiotherapy alone before surgery has not been described.

The review described by McCort [14] concluded that the prognosis is more favorable for carcinosarcoma than for SCC. Xu et al. [9] reported that the carcinomatous component of the tumor is usually at an early stage at the time of diagnosis and that the incidence of lymph node metastasis is thus low. However, the 5-year survival rates after treatment are similar to those reported for SCC [3, 4], since 5 years after surgery for carcinosarcoma, only $9(16.4 \%)$ of 55 patients remained alive and free of disease or had died of unrelated causes [3]. One study compared carcinosarcomas and SCC [4]. Although the 3-year survival rate was higher for carcinosarcoma than for SCC (62.8 vs. $28.1 \%$ ), the 5-year survival rates did not significantly differ between them (carcinosarcoma $26.7 \%$ vs. SCC $22.4 \%$ ) [4]. Thus, the prognosis is not as favorable as previously believed, due to the high possibility of hematogenous metastasis occurring later. Sanada et al. [6] reported that liver metastasis and peritoneal dissemination typically accompany sarcomatous components. The tumor in our patient was diagnosed as stage I at initial hospitalization and treated by neoadjuvant radiotherapy followed by total esophagectomy with regional lymph node dissection. Esophageal carcinosarcoma within stage I is generally treated by surgical resection, the same therapy as for esophageal SCC according to the Guidelines for Diagnosis and Treatment of Carcinoma of the Esophagus [15]. During initial hospitalization, the patient refused surgical treatment but accepted chemoradiotherapy. Due to renal dysfunction $(1.6 \mathrm{mg} / \mathrm{dl}$ of creatinine and $33.9 \mathrm{ml} / \mathrm{min}$ of estimated creatinine clearance), chemotherapy such as cisplatin plus 5-fluorouracil was considered to be unsuitable for the treatment of his esophageal carcinosarcoma. The patient declined the chemotherapy and provided informed consent to treatment with radiotherapy alone, which was not a standard therapy for esophageal carcinosarcoma based on the Guidelines for Diagnosis and Treatment of Carcinoma of the Esophagus [15]. Therefore, the patient was treated by neoadjuvant radiotherapy (40 Gy) without chemotherapy to reduce the tumor and improve his dysphagia, and then he finally accepted surgical treatment. However, he died 16 months later due to lung metastasis which we considered was caused by late hematogenous metastasis. The neoadjuvant radiotherapy ameliorated his symptom such as dysphagia and improved his quality of life although he finally died. The neoadjuvant radiotherapy for esophageal carcinosarcoma was considered to have contributed to the subsequent surgery and his prolonged survival time. 
Ogasawara et al.: Rapidly Growing Esophageal Carcinosarcoma Reduced by Neoadjuvant Radiotherapy Alone

In conclusion, we describe a rapidly growing esophageal carcinosarcoma that was reduced by radiotherapy alone before surgical treatment. Thus, radiotherapy alone might be a useful candidate for neoadjuvant therapy of esophageal carcinosarcomas.

\section{Disclosure Statement}

The authors have no conflict of interest.

\section{References}

1 Lee RG: Esophagus; in Sternberg SS (ed): Diagnostic Surgical Pathology, ed 3. Philadelphia. Lippincott Williams \& Wilkins 1999, pp 1283-1309.

-2 Geisinger KR, Dabbs DJ, Marshall RB: Malignant mixed müllerian tumors. An ultrastructural and immunohistochemical analysis with histogenetic considerations. Cancer 1987;59:1781-1790.

3 Iascone C, Barreca M: Carcinosarcoma and pseudosarcoma of the esophagus: two names, one disease comprehensive review of the literature. World J Surg 1999;23:153-157.

4 Iyomasa S, Kato H, Tachimori Y, Watanabe H, Yamaguchi H, Itabashi M: Carcinosarcoma of the esophagus: a twenty-case study. Jpn J Clin Oncol 1990;20:99-106.

5 Iwaya T, Maesawa C, Uesugi N, Kimura T, Ogasawara S, Ikeda K, Kimura Y, Mitomo S, Ishida K, Sato N, Saito K, Masuda T: True carcinosarcoma of the esophagus. Dis Esophagus 2006;19:48-52.

6 Sanada Y, Hihara J, Yoshida K, Yamaguchi Y: Esophageal carcinosarcoma with intramural metastasis. Dis Esophagus 2006;19:119-131.

7 The Japan Esophageal Society: Guidelines for Clinical and Pathologic Studies on Carcinoma of the Esophagus, ed 10. Tokyo, Kanehara Shuppan, 2007.

-8 Talbert JL, Cantrell JR: Clinical and pathologic characteristics of carcinosarcoma of the esophagus. J Thorac Cardiovasc Surg 1963;45:1-12.

-9 Xu LT, Sun CF, Wu LH, Chang ZR, Liu TH: Clinical and pathological characteristics of carcinosarcoma of the esophagus: report of four cases. Ann Thorac Surg 1984;37:197-203.

10 Virchow R: Vorlesungen über Pathologie: die krankhaften Geschwülste. Berlin, A. Hirschwald, 1865.

11 Ro JY, Chen JL, Lee JS, Sahin AA, Ordóñez NG, Ayala AG: Sarcomatoid carcinoma of the lung. Immunohistochemical and ultrastructural studies of 14 cases. Cancer 1992;69:376-386.

-12 Japanese Society for Esophageal Diseases: Guidelines for clinical and pathological studies on carcinoma of the esophagus, ninth edition (preface, general principles, part I). Esophagus 2004;1:61-88.

13 Yamamoto Y, Watanabe Y, Horiuchi A, Yoshida M, Yukumi S, Sato K, Nakagawa H, Sugishita H, Ishida N, Furuta S, Kawachi K: True carcinosarcoma of the esophagus: report of a case. Case Rep Gastroenterol 2008;2:330-337.

14 McCort JJ: Esophageal carcinosarcoma and pseudosarcoma. Radiology 1972;102:519-524.

15 Kuwano H, Nishimura Y, Ohtsu A, et al: Guidelines for diagnosis and treatment of carcinoma of the esophagus. April 2007 edition: Part I. Edited by the Japan Esophageal Society. Esophagus 2008;5:61-73. 
Ogasawara et al:: Rapidly Growing Esophageal Carcinosarcoma Reduced by Neoadjuvant Radiotherapy Alone
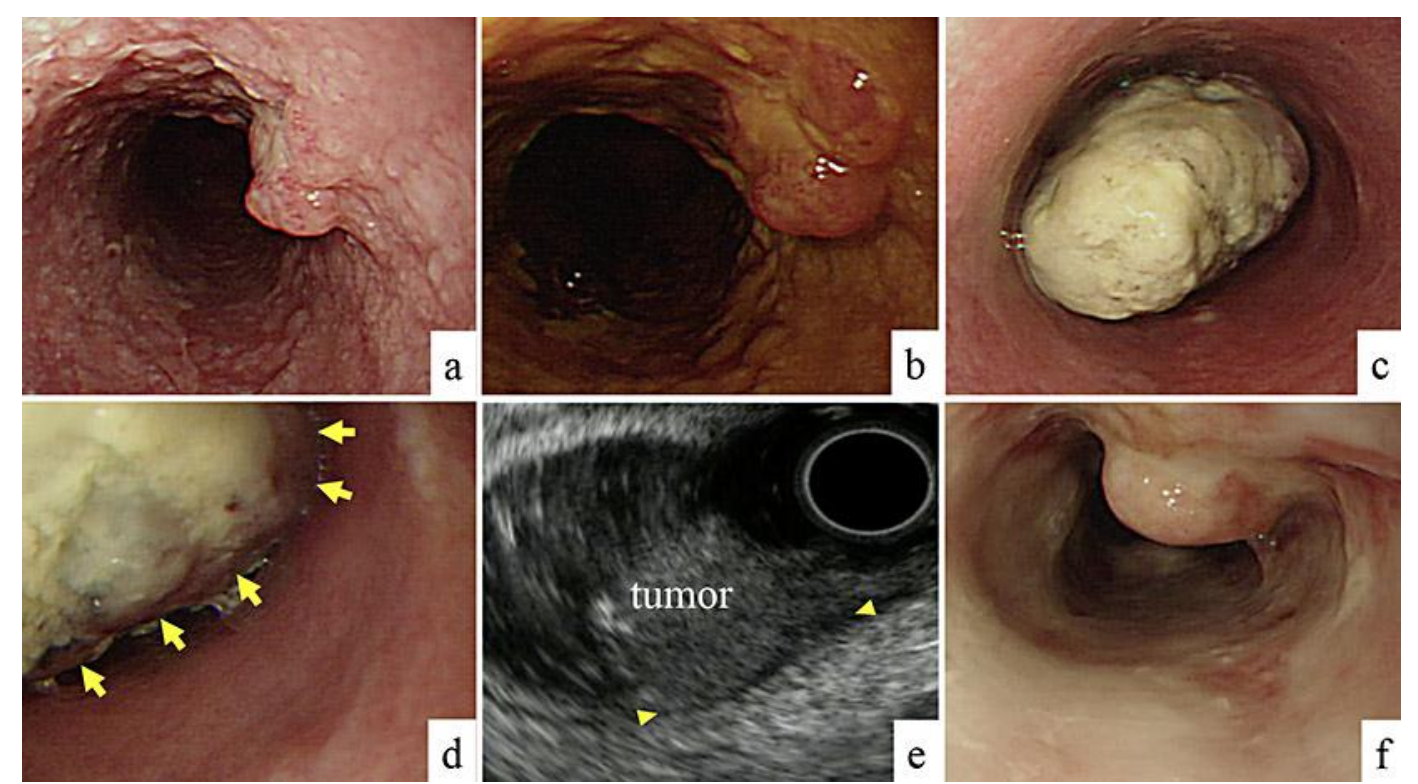

Fig. 1. Findings of first, second and third assessments by EGD. a The first EGD showed a small nodular polypoid lesion with central ulceration in the lower esophagus surrounded by rough esophageal epithelial mucosa. $\mathbf{b}$ The tumor surface was not stained with Lugol's iodine due to possible gastroesophageal reflux of stomach contents. c The second EGD 1 month later showed a huge mass with a relatively smooth and fuzzy surface occupying the esophageal lumen. $\mathbf{d}$ The tumor was partially covered with normal esophageal mucosa (arrows). e Esophageal ultrasonography showed the tumor to be mainly localized within the submucosal layer. Internally, the hypoechoic tumor had a relatively homogeneous pattern. The muscularis propria was apparent (arrowheads). $f$ The third EGD showed that the tumor was remarkably reduced to a small polypoid lesion 1 month after 40 Gy of radiotherapy. 

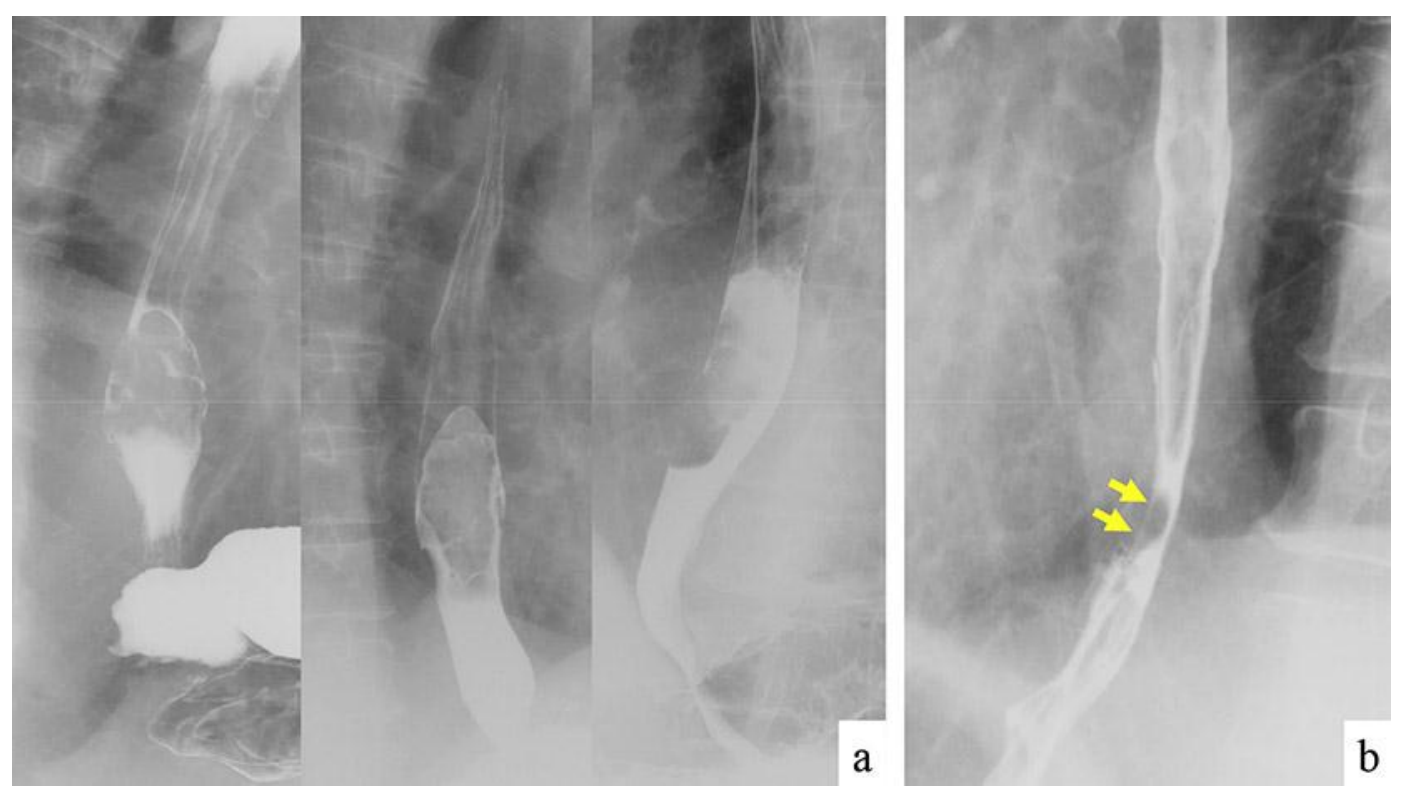

Fig. 2. Barium esophagography findings of the lesion before and after 40 Gy of radiotherapy. a Image showing a lobulated polypoid lesion about $6 \mathrm{~cm}$ in diameter with a relatively smooth surface, central superficial irregularity and depression in the lower esophagus. b Barium esophagography after 40 Gy of radiotherapy showed a tumor about $1 \mathrm{~cm}$ in diameter with a smooth surface (arrows). 
Ogasawara et al.: Rapidly Growing Esophageal Carcinosarcoma Reduced by Neoadjuvant Radiotherapy Alone
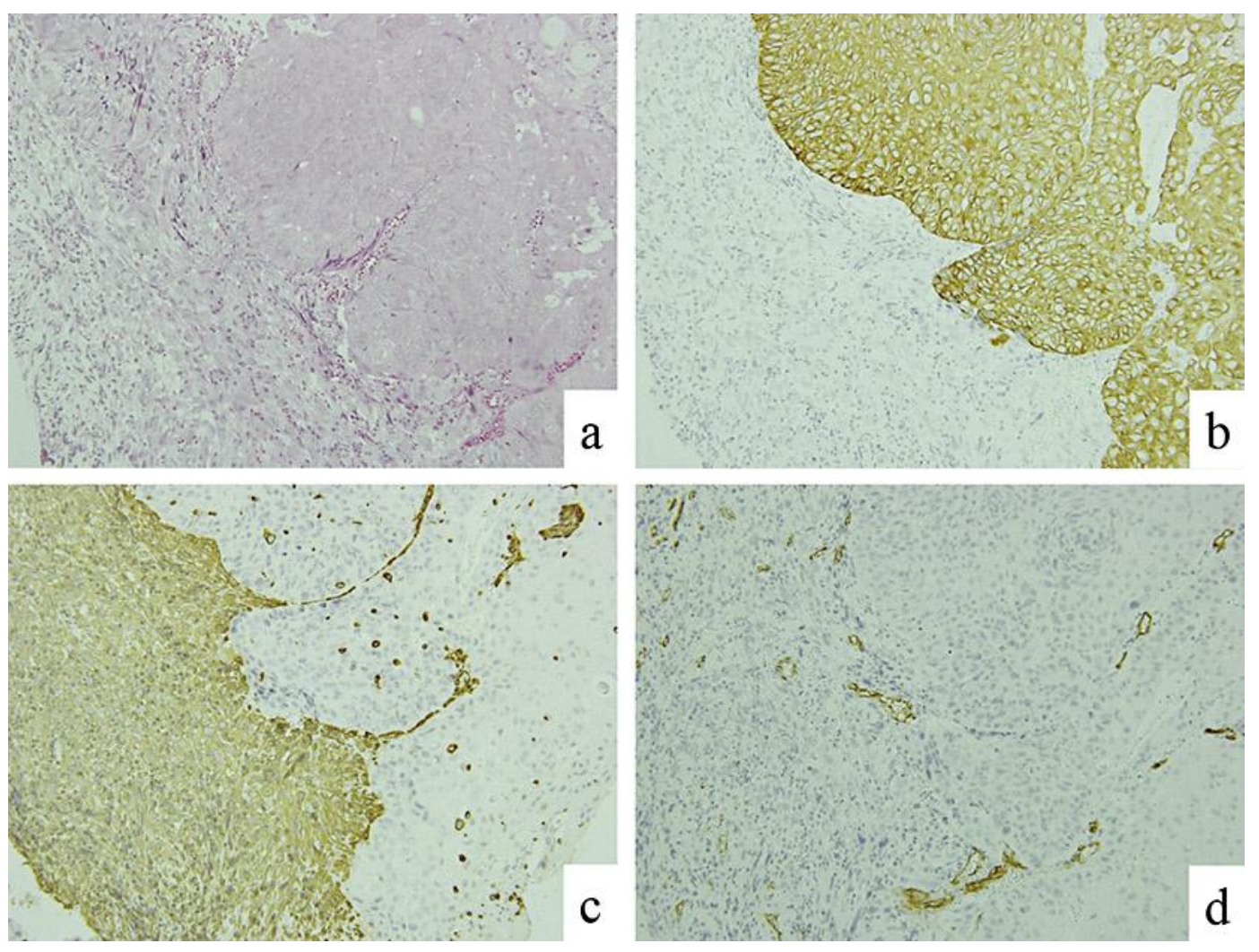

Fig. 3. Immunohistochemical findings of a tumor biopsy specimen. a Hematoxylin and eosin staining showed the tumor to be comprising poorly differentiated SCC with spindle cell components and transitional features between them. The SCC (b) and spindle-shaped tumor cells (c) were immunohistochemically positive for cytokeratin and vimentin, respectively. Both SCC and spindle-shaped tumor cells were negative for CD34 (d). Original magnification $\times 200$. 